\title{
WORLD HEALTH ORGANIZATION
}

EXECUTIVE BOARD

EB115/39

115th Session

Provisional agenda item 4.13

16 December 2004

\section{eHealth}

\section{Report by the Secretariat}

\section{BACKGROUND}

1. The emergence and growth of information and communication technologies, touching many spheres of life, have in the past decade brought opportunity and challenge to all countries. This is reflected in the Millennium Development Goals, especially target 18: "In cooperation with the private sector, make available the benefits of new technologies, especially information and communications".

2. WHO has for many years undertaken activities on the use of information technology for health care and medical purposes. For example, an international consultation convened by WHO (December 1997) prepared input on "telematics" for WHO's health-for-all policy for the twenty-first century. ${ }^{1}$ Resolution WHA51.9 (1998) sets out lines of action in relation to cross-border advertising, promotion and sale of medical products through the Internet.

3. Today, eHealth - understood in this context to mean use of information and communication technologies locally and at a distance - presents a unique opportunity for the development of public health. The strengthening of health systems through eHealth reinforces fundamental human rights by improving equity, solidarity, quality of life and quality of care.

4. Member States and groups of Members States are drafting their own strategies for eHealth, and other organizations of the United Nations system have drawn up strategies for information and communication technologies in their domains. eHealth was one of the topics discussed at the World Summit on the Information Society (Geneva, December 2003).

5. In view of the foregoing, WHO's Secretariat has prepared a draft strategy for eHealth that would serve as the basis for coordinating both eHealth policies internationally and WHO's activities on eHealth. ${ }^{2}$ It sets the general direction for work in this area, together with specific lines of action based on WHO's goals and objectives. It indicates ways of providing support to Member States in the use of eHealth for public-health purposes, health-care delivery, capacity building, and governance. It also includes eLearning for the public, meaning, in this context, the use of any electronic technology and media in the support of learning.

\footnotetext{
${ }^{1}$ See document EB101/INF.DOC./9.

${ }^{2}$ See document EGB/eHealth No.1, available in the Executive Board room.
} 


\section{POTENTIAL IMPACT ON HEALTH}

6. Extremely rapid changes are occurring because of the advances in information and communication technologies. eHealth may be expressed in terms of digitalized products, systems, and services for health. These technologies hold great promise for both low- and high-income countries, and some are already realizing the benefits. These benefits apply not only to health-care delivery, but also to public health, governance, finance, education, research, and health-related economic activities.

7. eHealth should have an impact on health systems by making health services more efficient and improving access to care, especially in remote areas, for people with disabilities and for the elderly. It should benefit health-care providers, professionals, and final users through higher quality of care and health promotion. It should also affect the cost of care by reducing redundancy and duplication of examinations and making possible economies of scale.

\section{GLOBAL AND NATIONAL CHALLENGES}

8. There are many partners involved in health who are not only the beneficiaries of, but also a driving force for, use of information and communication technologies in Member States. Thus, they should participate actively in international forums for collaboration on information and communication technologies so that their viewpoints are represented. Flow of health data no longer has any barriers. Although this situation needs regulating, it is also an opportunity for faster and more comprehensive epidemiological surveillance. A global approach to handling data flows will help to promote standardization and low-cost services.

9. Technological excellence is growing in low-income countries, which are developing their own expertise. However, for many the benefits expected of eHealth have not yet materialized, and it is difficult to predict the rate and extent to which information and communication technologies will affect diverse health systems. It is essential to evaluate and share experience in order to develop individualized cost-effective models and, in particular, to understand the determinants involved in the adoption and sustainability of eHealth. Prerequisites for the successful integration of eHealth into health-care systems include long-term government commitment, based on a strategic plan, national awareness of the benefits of eHealth, and availability of skilled human resources.

\section{eHEALTH, RIGHTS AND ETHICS}

10. Inequalities exist in access to, and use of, information and communication technologies, within and between countries. Human, ethical, and legal rights issues are also involved, in particular the right to the highest attainable standard of health.

11. Efforts are needed to tackle the undue burden of ill-health borne by vulnerable and marginalized groups. It is essential to invest in development of infrastructure for information and communication technologies for health, and to ensure equitable, affordable, and universal access to their benefits.

12. Ethical issues concern all countries in respect of confidentiality of information, dignity, and privacy. Respect for the principles of equity is vital, considering differences in culture, education, language, geographical location, physical and mental ability, age and sex. Information and communication technologies should maximize the use of scarce resources, rather than divert resources from meeting people's basic health needs. 
13. eHealth issues pose new legal challenges. Many applications of eHealth are currently unregulated, unlike other aspects of the health systems. Legislation governing confidentiality, privacy, access, and liability is necessary with the transfer of information internally and externally. There is a growing interdependence and closer collaboration between the public sector and the private and nonprofit sectors in information and communication technologies. Although public and private partnerships are welcome, the maintenance of minimum public services for health must be guaranteed.

\section{OPPORTUNITY FOR WHO}

14. As the United Nations specialized agency for health, WHO has a constitutional role to act as the directing and coordinating authority on international health work. It can thus engage in, and serve as convenor for, any area or endeavour that can be of benefit to global health. As such, it can provide an interface between external public and private bodies, draw up relevant standards and guidelines, and develop methods for assessment.

15. Advances in information and communication technologies have raised expectations of Member States, partners, and other international organizations, which provides an opportunity for WHO to respond to demands and to be a driving force for active collaboration.

\section{COMPONENTS OF WHO'S DRAFT EHEALTH STRATEGY}

16. WHO's Secretariat has drawn up a draft strategy focusing on equity and quality, and based on the principles of the Charter of the United Nations. The main lines are:

- to define a set of priority activities based on health needs to be supported and promoted at global, regional and national levels

- to disseminate widely eHealth experiences and best practices

- to strengthen the ability of Member States to address health problems through the integration of eHealth applications into health systems

- to provide support to Member States for establishing safe and reliable eHealth applications through the adoption of sets of basic requirements for services covering policy, quality, safety, access and use

- to prepare norms, standards, guidelines, and information and training materials

- to provide support for development of methodologies for assessing eHealth products and services

- to "speak with one voice" on issues that will impact the equitable development of eHealth in countries and globally

- to promote research and development of eHealth for the benefit of Member States

- to foster exchange of data and information for promotion of health, health systems, and training of health-care workers 
- to provide to countries sound technical and policy support and guidance on best practice and implementation tools and methods

- to investigate, analyse, and document the impact on the health of populations of policies and interventions related to information and communication technologies, including social and economic.

\section{FRAMEWORK FOR ACTION}

17. To put the foregoing into effect, Member States need to formulate cost-effective eHealth strategies reflecting principles of transparency, ethics, and equity. This implies collaboration with sectors other than health in order to develop infrastructure and to strengthen information and communication technologies for public health, health-care delivery, health education and training, within a multilingual and multicultural approach.

18. Transparent, equitable and ethical public-private partnerships may have to be created in order to gain access to resources for improving eHealth content, and to build capacity and infrastructure. Monitoring of achievements towards internationally agreed targets and goals related to eHealth could be achieved through collaboration with other organizations of the United Nations system and partners.

19. Member States will need to ensure that eHealth for citizens, patients and health professionals meets quality, safety and ethical standards. This may be supported by mobilizing multisectoral collaboration for determining evidence-based eHealth standards and norms. Further, best use should be made of eHealth for sharing health information between health-care professionals, for collecting data, including from sentinel systems, controlling quality and identifying best practices for prevention.

20. WHO's Secretariat intends to establish a facility - a global eHealth observatory - to document and analyse developments and trends, inform policy and practice in countries, and report regularly on use of eHealth worldwide. National centres and networks of excellence in eHealth will need to be established in order to identify eHealth best practice, coordinate policy, provide technical support, deliver health care, improve services, inform citizens, build capacity and undertake surveillance.

21. Other actions include extension of such mechanisms as the Health Academy to and within Member States in order to promote awareness of health and healthy lifestyles through eLearning; analyse the evolution of eHealth and its impact on health; anticipate emerging challenges and opportunities; and provide evidence, information, and guidance in support of policy, best practice, and management of eHealth services.

\section{ACTION BY THE EXECUTIVE BOARD}

22. The Board is invited to consider the following draft resolution: 
The Executive Board,

Having considered the report on eHealth, ${ }^{1}$

RECOMMENDS to the Fifty-eighth World Health Assembly, the adoption of the following resolution:

The Fifty-eighth World Health Assembly,

Having considered the report on eHealth;

Noting the potential impact that advances in information and communication technologies could have on health-care delivery, public health, governance, finance, education, research and health-related economic activities for the benefit of both low- and high-income countries;

Aware that advances in information and communication technologies have raised expectations for health on the part of Member States of WHO, partners and other international organizations;

Respecting the principles of equity, considering differences in culture, education, language, geographical location, physical and mental ability, age, and sex;

Recognizing that a WHO eHealth strategy would serve as a basis for developing both eHealth policies internationally and WHO's activities on eHealth;

Recalling resolution WHA51.9 on cross-border advertising, promotion, and sale of medical products through the Internet;

Noting the work already done on developing a draft strategy on eHealth for WHO,

1. URGES Member States:

(1) to consider drawing up a long-term strategic plan for developing and implementing eHealth services; ${ }^{2}$

(2) to invest in the development of infrastructure for information and communication technologies for health, and to ensure equitable, affordable, and universal access to their benefits;

(3) to consider legislation governing confidentiality, privacy, access and liability with respect to the transfer of information internally and externally across borders;

\footnotetext{
${ }^{1}$ Document EB115/39.

2 eHealth is understood in this context to mean use of any information and communication technologies locally and at a distance.
} 
(4) to build on closer collaboration with the private and non-profit sectors in information and communication technologies, while guaranteeing maintenance of public services for health;

(5) to endeavour to reach communities, including vulnerable groups, with eHealth services appropriate to their needs;

(6) to encourage public-private partnerships and to accelerate capacity building in order to improve access to eHealth resources by institutions, professionals and citizens;

(7) to mobilize multisectoral collaboration for determining evidence-based eHealth standards and norms, and to ensure that eHealth for citizens, patients and health professionals meet quality, safety, and ethical standards;

(8) to evaluate eHealth activities and to share knowledge with a view to developing individualized cost-effective models, making full use of existing experience;

(9) to establish national centres and networks of excellence for eHealth best practice, policy coordination, and technical support for health-care delivery, service improvement, information to citizens, capacity building, and surveillance;

2. REQUESTS the Director-General:

(1) to promote international, multisectoral collaboration with a view to improving compatibility of administrative and technical solutions in the area of eHealth;

(2) to document and analyse developments and trends, inform policy and practice in countries, and report regularly on use of eHealth worldwide;

(3) to provide technical support to Member States in relation to eHealth products and services by disseminating widely experiences and best practices; devising assessment methodologies; promoting research and development; and furthering standards through diffusion of guidelines;

(4) to facilitate the integration of eHealth in health systems and services, including in the training of health-care professionals and in capacity building, in order to improve access to, and quality and safety of, care;

(5) to continue the expansion to Member States of mechanisms such as the Health Academy which promote health awareness and healthy lifestyles through eLearning. ${ }^{1}$

$$
=\quad=
$$

${ }^{1}$ eLearning is understood in this context to mean use of any electronic technology and media in support of learning. 